\title{
The role of color-magnetic monopoles in a gluon plasma
}

\author{
Claudia Ratti ${ }^{* a, b}$, Edward Shuryak ${ }^{b}$ \\ ${ }^{a}$ Department of Theoretical Physics, University of Wuppertal, Wuppertal 42119, Germany \\ ${ }^{b}$ Department of Physics \& Astronomy, SUNY Stony Brook, NY 11794-3800, USA. \\ E-mail: cratti@ph.tum.de
}

The role of color-magnetic monopoles in a pure gauge plasma at high temperature $T>2 T_{c}$ is considered. In this temperature regime, monopoles can be considered heavy, rare objects embedded into matter consisting mostly of the usual "electric" quasiparticles, quarks and gluons. The gluon-monopole scattering is found to hardly influence thermodynamic quantities, yet it produces a large transport cross section, significantly exceeding that for pQCD gluon-gluon scattering up to quite high $T$. This mechanism keeps viscosity small enough for hydrodynamics to work at LHC.

International Workshop on QCD Green's Functions, Confinement, and Phenomenology - QCD-TNT09 September 07 - 112009

ECT Trento, Italy

\footnotetext{
*Speaker.
} 


\section{Introduction}

Creating and studying Quark-Gluon Plasma (QGP) in the laboratory has been the goal of experiments at CERN SPS and recently at the Relativistic Heavy Ion Collider (RHIC) facility in Brookhaven National Laboratory, soon to be continued by the ALICE collaboration at the Large Hadron Collider (LHC). RHIC experiments have revealed robust collective phenomena in the form of radial and elliptic flows, which turned out to be quite accurately described by near-ideal hydrodynamics. QGP thus seems to be the most perfect liquid known, with the smallest viscosity-to-entropy ratio $\eta / s$. One of the central questions is how sQGP with "near-perfect fluidity" will change into a weakly coupled wQGP with increasing $T$. In view of the next round of heavy ion experiments at LHC, a quite urgent question is what transport properties are expected to be observed there, at temperatures reaching about twice those reached at RHIC. In order to answer this question, one of course has to understand where the "perfect fluidity" property of QGP comes from.

Recently, the electric - magnetic duality has been proposed, and used to explain unusual properties of the QGP [1]: in this so-called "magnetic scenario", the near- $T_{c}$ region is dominated by magnetic monopoles. Another line of work based on lattice monopoles has led Chernodub and Zakharov [2] at the same time to a very similar conclusion. An important feature of this scenario is the opposite running of the electric coupling $e$ and the magnetic one $g$, induced by the Dirac condition $e g=$ const. As recently shown in [3], this feature has been dramatically confirmed by the behavior of the lattice correlation functions [4], which indeed display monopole-monopole and antimonopole-monopole correlations increasing with $T$. As shown in [3], those correlations are well described by a picture of classical Coulomb gas. The main input in those calculations is the magnetic Coulomb coupling g growing with $T$. Furthermore, it was shown to be simply the inverse of the gauge coupling $e$, as Dirac predicted. We consider the correlations observed in [4] to be a decisive confirmation of the existence of the long-distance magnetic Coulomb field of the monopoles. It is natural to investigate the role played by these objects in the QGP [5, 6, 7]: we therefore address here the issue of QGP transport properties in the "magnetic scenario" framework. We move away from the phase transition region to higher temperatures, where QGP is still dominated by the usual electric quasiparticles - quarks and gluons - and the coupling is moderately small. Our goal is to study the interaction between electric and magnetic sectors. Our main result is the explicit solution of the problem of quantum gluon-monopole scattering, from which we calculate the corresponding transport cross sections. This will allow us to get a temperature-dependent estimate of the ratio $\eta / s$.

\section{Brief review of lattice monopoles}

Lattice monopoles are defined by the procedure [8] which basically locates the ends of singular Dirac strings by calculating the total magnetic flux through the boundary of elementary 3-d boxes. Since the strings and thus the procedure depend on a certain gauge, for decades sceptics kept the viewpoint that those objects are just unphysical UV gauge noise. Yet, many specific questions e.g. monopole density and correlations [4] - produced very reasonable and consistent answers, which are apparently independent of the particular lattice parameters. It is hard to imagine that lattice gauge artifacts have consistent correlations at relatively large distances, accurately repro- 
ducing those in a Coulomb plasma. These recent results strongly support the assumption which was first made in ref. [1], according to which monopoles are meaningful physical degrees of freedom, present in the QGP as quasiparticles and being the source of a Coulomb-like magnetic field.

In Fig. 1 we summarize what is known about monopoles in the deconfined phase. The diamonds of different colors show the direct lattice observation of monopole density by D'Alessandro and D'Elia [4], scaled up by the factor 2 which accounts for the transition from SU(2) to SU(3) gauge group (in $\mathrm{SU}(2)$ there is one monopole species, in $\mathrm{SU}(3)$ there are two, identified by two different $U(1)$ subgroups). The best fit to the data of D'Alessandro and D'Elia (not shown) is

$$
n_{m} / T^{3}=A / \log \left(T / \Lambda_{\text {eff }}\right)^{\alpha}
$$

with $A=0.48, \alpha=1.89$ and $T_{c} / \Lambda_{\text {eff }}=2.48$.

The crosses in Fig. 1 are from Ref. [9]. They have been obtained from (metastable) flux tubes in the plasma phase. The latter should be compared to the solid line, which represents gluons, affected by the nonzero VEV of the Polyakov line effects ${ }^{1}$ [10]. (At high $T$ the normalized gluon density becomes a constant corresponding to Stefan-Boltzmann ideal gas of massless SU(3) gluons.) Note that the densities of gluons and monopoles cross each other at $T \simeq 1.3 T_{c}$. We also point out that crosses and diamonds in Fig. 1 show only a qualitative agreement, but do not match. This issue is partly due to the uncertainties in the extrapolation from $N_{c}=2$ (diamonds) to $N_{c}=3$ (crosses).

The available lattice results on the internal monopole structure are not too detailed. Fig. 2 is taken from the talk given by Ilgenfritz at Lattice 2007 [11]. Panel (a) and panel (b) show the structure, transversal to a monopole world line, of the "non-Abelianicity" (squared gauge potential not in the Abelian direction) and of the modulus of the topological density ${ }^{2}$, respectively. These two quantities are definitely correlated with the monopole current, however they display different sizes. The non-Abelianicity shows a narrower peak, with a small width of only about

$$
r_{m} \approx 1.5 a \approx 0.15 \mathrm{fm}
$$

(The lattice spacing was $a=0.105 \mathrm{fm}$ in this simulation). It is hardly surprising that we don't have any real insights about the monopole structure, as their size is so close to $a$ (the UV cutoff) of the best lattice calculations.

\section{Quantum gluon-monopole scattering}

We set up the problem of quantum scattering on monopoles in the framework of the GeorgiGlashow model [12], in which we perform the calculations presented in the present section. This is done because within this model it is possible to construct a monopole solution explicitly. The generalization to QCD will be discussed in the following. However, as the reader will see, the results that we find are quite general and don't depend on the specific, model-dependent structure

\footnotetext{
${ }^{1}$ The reader is reminded that it reflects the nonzero VEV of $A_{0}$, which acts on electrically charged particles as an imaginary chemical potential.

${ }^{2}$ Provided by the unfiltered overlap-defined topological density.
} 


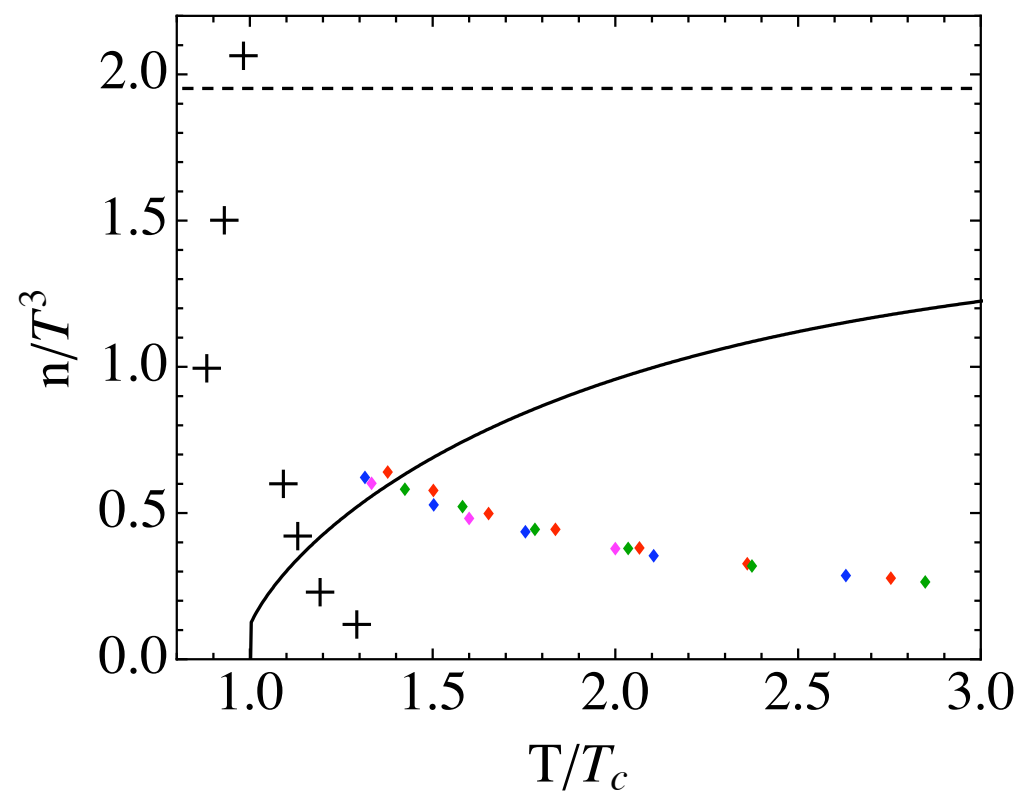

Figure 1: The normalized density $n / T^{3}$ versus $T / T_{c}$ for the deconfined phase $T>T_{c}$. Diamonds are lattice results taken from Ref. [4], while crosses are from Ref. [9]. The solid line represents the gluon density.

of the monopole core. The Georgi-Glashow model describes the interaction between gauge fields (two massive, spin-1 Ws and a massless photon) and a scalar Higgs field.

The wave function in spherical coordinates is the usual sum of products of certain $r$-dependent radial functions, times the angular functions. The former basically follow from the inverse-square law potential and thus are easily solved in Bessel functions. The nontrivial part happens to be in the unusual angular functions. Before introducing those, let us hint why the usual set of angular harmonics $Y_{l m}(\theta, \varphi)$ should not to be used. The reason is that their classical limit - for large values of the indices $m \approx l \gg 1$ - corresponds to flat planar motion near the $z=0$ plane. Nevertheless, from the classical limit we expect the motion to be concetrated around the Poincaré cone instead.

We denote the vector harmonics by $\Phi_{j, n}^{m, \sigma}(\theta, \varphi)_{a i}$. They obey the following eigenvalue equations

$$
\left\{\begin{array}{l}
\vec{J}^{2} \\
J_{3} \\
(\hat{r} \cdot \vec{I}) \\
(\hat{r} \cdot \vec{S})
\end{array}\right\} \Phi_{j, n}^{m, \sigma}(\theta, \varphi)_{a i}=\left\{\begin{array}{l}
j(j+1) \\
m \\
n \\
\sigma
\end{array}\right\} \Phi_{j, n}^{m, \sigma}(\theta, \varphi)_{a i}
$$

where $\vec{J}$ is the total angular momentum $\vec{J}=\vec{L}+\vec{I}+\vec{S}=\vec{T}+\vec{S}, \vec{L}$ is the orbital angular momentum, $\vec{I}$ the isospin and $\vec{S}$ the spin. The unusual conditions in the above set are the last two, since the vectors $\vec{I}$ and $\vec{S}$ must be projected to the (space-dependent) radial unit vector.

The problem of quantum gluon-monopole scattering is solved in the pointlike monopole approximation. This is justified by the information on the monopole size that we obtained from Fig. 2. In this case, and for $j \neq 0$, the equations for the radial functions $T_{j \alpha}(\xi)$ reduce to generalized Bessel-like equations with noninteger index $j^{\prime}=-\frac{1}{2}\left[-1+\sqrt{(2 j+1)^{2}-4 n^{2}}\right]$, where $n=e g$ is the product of electric and magnetic couplings and $j$ is the total angular momentum quantum 


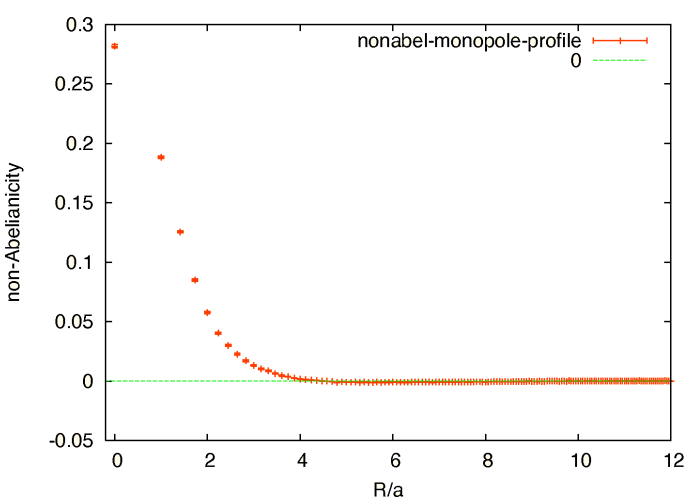

(a)

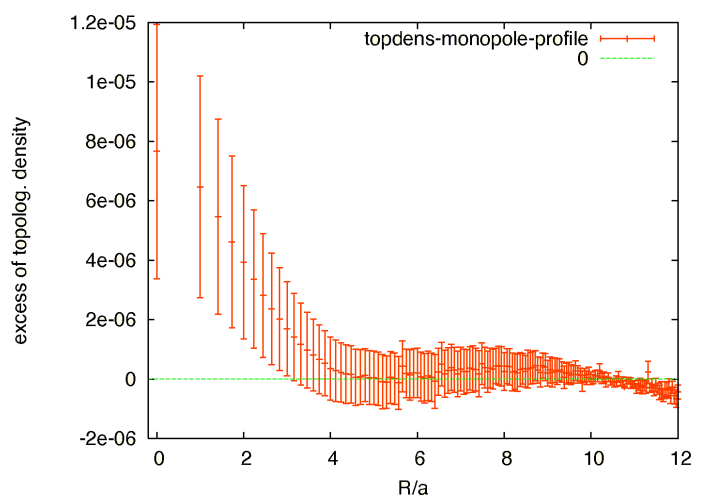

(b)

Figure 2: The square of non-Abelian components of the gauge potentials (a) and the topological charge (b) correlated with the monopole path (taken from Ref. [11]).

number:

$$
T_{j \alpha}^{\prime \prime}(\xi)-\left[-\omega^{2}+1+\frac{j(j+1)-n^{2}}{\xi^{2}}\right] T_{j \alpha}(\xi)=0 .
$$

The index $\alpha$ runs from 0 to 9 and indicates all possible combinations of charge and spin polarization for gluons. After gauge fixing, three combinations turn out to be unphysical, and only six survive.

The scattering phase that we obtain from Eq. (3.2) is $\delta_{j^{\prime}}=-j^{\prime} \frac{\pi}{2}$, independent of energy. This feature is very important, since the contribution of this kind of scattering to thermodynamics is given by the Beth-Uhlenbeck formula

$$
\delta M_{m}=\frac{T}{\pi} \sum_{j}(2 j+1) \int d k \frac{d \delta_{j}}{d k} f(k, T)
$$

which vanishes identically for a constant scattering phase. Therefore, we find that the gluonmonopole scattering does not contribute to thermodynamics. There is an exception to this result, for $j=0$. In this case, the gluon can penetrate the monopole core and form bound states. We don't discuss this case here, for all details we refer the reader to Ref. [5].

\section{Transport cross section: results}

The scattering amplitude $f(\theta)$ is given by the following formula:

$$
2 i k f(\theta)_{n, v}=\sum_{j=|v|}^{j_{\max }}(2 j+1) e^{i \pi\left(j^{\prime}-j\right)} d_{v,-v}^{(j)}(\theta)
$$

where $v=n+\sigma=(\vec{T} \cdot \hat{r})+(\vec{S} \cdot \hat{r})=-J_{3}$. The sum over $j$ has an upper cutoff $j_{\text {max }}$ : in matter there is a finite density of monopoles, so the issue of the scattering should be reconsidered. A sketch of 


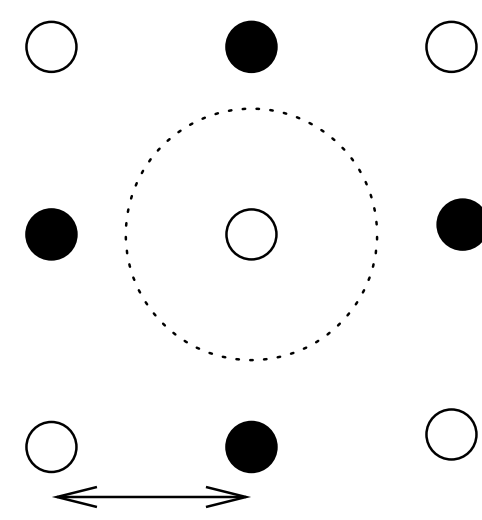

a

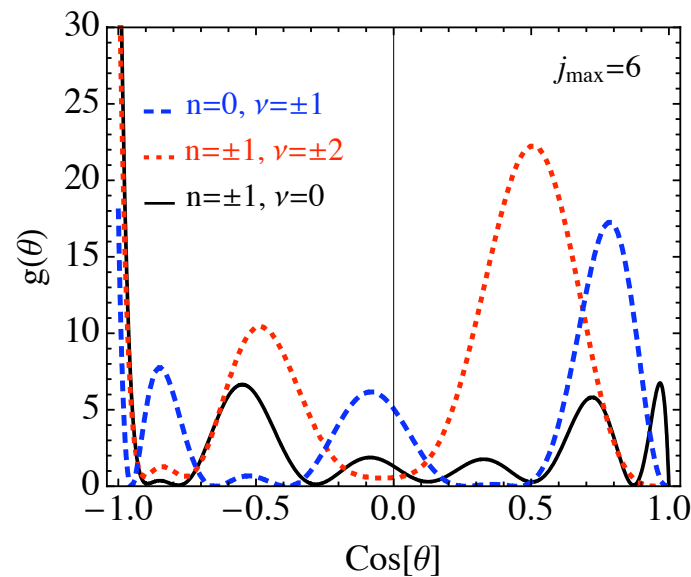

Figure 3: Left: A charge scattering on a 2-dimensional array of correlated monopoles (open points) and antimonopoles (closed points). The dotted circle indicates a region of impact parameters for which scattering on a single monopole is a reasonable approximation. Right: Integrand of the transport cross section $g(\theta)=$ $(1-\cos (\theta))|f(\theta)|^{2}$ with only 6 lowest partial waves included, for a gluon with $n=0, v= \pm 1, n= \pm 1, v=$ 0 and $n= \pm 1, v= \pm 2$. The strong peak backwards is due to the presence of the cutoff $j_{\max }$.

the setting, assuming strong correlation of monopoles into a crystal-like structure, is shown in the left panel of Fig. 3. A "sphere of influence of one monopole" (the dotted circle) gives the maximal impact parameter to be used. As a result, the impact parameter is limited from above by some $b_{\max }$, which implies that only a finite number of partial waves should be included. The range of partial waves to be included in the scattering amplitude can be estimated as follows

$$
j_{\max }=\left\langle p_{x}\right\rangle n_{m}^{-1 / 3} / 2 \sim a T \sim 1 / e^{2}(T) \sim \log (T) .
$$

Since at asymptotically high $T$ the monopole density $n_{m} \sim\left(e^{2} T\right)^{3}$ is small compared to the density of quarks and gluons $\sim T^{3}, j_{\max }$ asymptotically grows logarithmically with $T$. So, only in the academic limit $T \rightarrow \infty$ one gets $j_{\max } \rightarrow \infty$ and the usual free-space scattering amplitudes calculated in [13] where all partial waves are recovered. However, in reality we have to recalculate the scattering, retaining only several lowest partial waves from the sum. Taking the lattice results on the monopole density as a function of the temperature [4], we estimate $j_{\max } \simeq 6$ in our temperature regime. This dramatically changes the angular distribution, by strongly depleting scattering at small angles and enhancing the scattering backwards. This is evident in the right panel of Fig. 3, where we show the angular distribution of the integrand of the transport cross section $\sigma_{t}$ :

$$
\left(\sigma_{t}\right)_{n, v}=\int_{-1}^{1} d \cos \theta(1-\cos \theta)\left|f(\theta)_{n, v}\right|^{2} .
$$

The integrand exhibits a strong peak backwards, which would disappear in the absence of $j_{\max }$.

We now proceed to evaluate the scattering rate of gluons on monopoles:

$$
\frac{\dot{w}_{g m}}{T}=\frac{\left\langle n_{m}\left(\sigma_{t}\right)_{g m}\right\rangle}{T}
$$



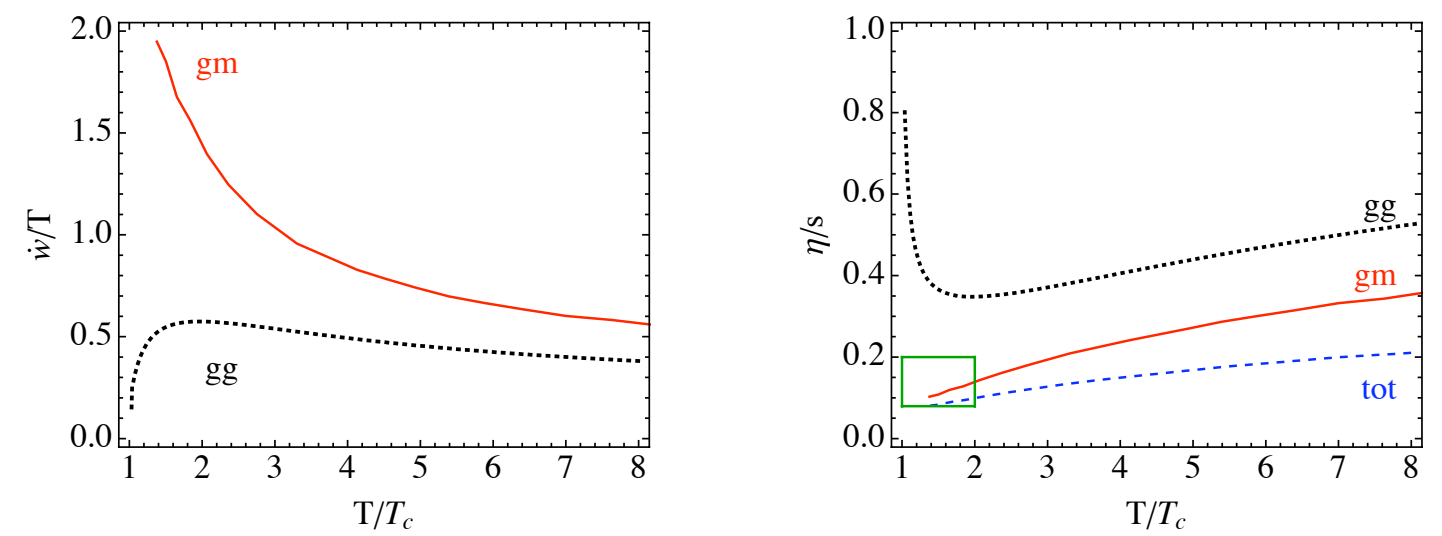

Figure 4: Left panel: gluon-monopole and gluon-gluon scattering rate. Right panel: gluon-monopole and gluon-gluon viscosity over entropy ratio, $\eta / s$. The blue, dashed curve is the total $\eta / s$, which is evaluated from the $g g$ and $g m$ contributions. The green box represents the present estimate of $\eta / s$ in the RHIC temperature regime.

where the $\langle\ldots\rangle$ indicates an average over the incoming gluon. $n_{m}$ is taken from Ref. [4] and is $n_{m} \simeq 0.02 \mathrm{GeV}^{3}$ in our temperature regime (see Fig. 1). The gluon density has the following form:

$$
\begin{aligned}
n_{g}(T) & =\frac{8 \pi}{(2 \pi)^{3}} \int k^{2} d k\left[\frac{2}{\exp \left(\beta \varepsilon_{k}\right)-1}+\frac{2}{\exp \left(\beta \varepsilon_{k}\right) \exp \left(i \beta \mathscr{A}_{0}^{3}\right)-1}+\frac{2}{\exp \left(\beta \varepsilon_{k}\right) \exp \left(-i \beta \mathscr{A}_{0}^{3}\right)-1}\right. \\
& \left.+\frac{1}{\exp \left(\beta \varepsilon_{k}\right) \exp \left(2 i \beta \mathscr{A}_{0}^{3}\right)-1}+\frac{1}{\exp \left(\beta \varepsilon_{k}\right) \exp \left(-2 i \beta \mathscr{A}_{0}^{3}\right)-1}\right]=\frac{4 \pi}{(2 \pi)^{3}} \int k^{2} d k \rho_{g}(k, T)(4.5)
\end{aligned}
$$

where we have taken into account the suppression of electric particles due to the coupling with the Polyakov loop (see for example [14]): $\mathscr{A}_{0}^{3}$ is a temporal background gauge field related to the Polyakov loop. In the average over the incoming gluon, we have to take this suppression into account by integrating over $\vec{k}$ with the weight $\rho_{g}(k, T)$. We show $\dot{w}_{g m} / T$ in the left panel of Fig. 4 (the red, continuous line). Also shown is the same quantity for the $g g$ scattering process (black, dotted line). The approximate relation of the scattering rate to viscosity/entropy ratio is $(\eta / s) \approx(T / 5 \dot{w})$. We plot $\eta / s$ in the right panel of Fig. 4 .

\section{Conclusions and discussion}

In this paper we studied the role of magnetic monopoles in the "electric" QGP phase. In distinction with papers by Liao and Shuryak, we have not focused on the near- $T_{c}$ region, in which monopoles seem to be dominant over gluons in number, and may even expel electric fields into flux tubes as they do in the confined phase. Instead, we considered the more traditional QGP away from $T_{c}$, dominated by the usual "electric" constituents - quarks and gluons, the magnetic monopoles being subleading in number.

More specifically, we focused on scalar-monopole and gluon-monopole scattering. Classical molecular dynamics, studied in refs. [1], suggested a very interesting mechanism of " "mutual trapping" by electric/magnetic quasiparticles. Based on results of quantum scattering on monopoles $[13,15,16]$ for scalar-monopole and spinor-monopole scatterings, in this paper we studied this effect further. Indeed, we found significant large angle and even backward scattering for small 
impact parameters, complemented with a Rutherford-like forward scattering peak. Our main result is a detailed derivation of the gluon-monopole scattering amplitude, especially in the lowest partial waves. For gauge particles, the existence of spin and isospin leads to complications which so far precluded this problem from being solved. Our first important finding is that the gluon-monopole scattering has little effect on thermodynamics, because scattering phases - albeit large - are mostly energy independent, except the "exceptional channel" with $j=0$. This explains why lattice studies which focused on thermodynamic observables were unable to see such effects. Our second (and the main) finding is that the contribution of gluon-monopole scattering is very important for transport properties. While the monopole density may be small, the $g m$ scattering amplitudes have $e^{2} g^{2} \sim$ $O(1)$ coupling instead of small $e^{4} \ll 1$. Furthermore, in our setting (with a limited number of partial waves $j<j_{\max }$ included) there is an additional enhancement for large angle (or even backward) scattering. Convoluting the cross sections found with the monopole density and gluon momentum distribution, we plot the scattering rates $n \sigma_{t}$ per gluon vs $T$ in Fig.4.

It follows from this comparison of the gluon-monopole curve with the gluon-gluon one that the former remains the leading effect till very high $T$, although asymptotically it is expected to get subleading. The maximal $T$ expected at LHC does not exceed $4 T_{c}$, where the total $\eta / s \sim .15$. This value is well in the region which would ensure hydrodynamical radial and elliptic flows, although deviations from ideal hydro would be larger than at RHIC (and measurable!).

We plot $\eta / s$ in the right panel of Fig. 4. We observe a qualitative agreement between our results and the experimental value for $\eta / s$ observed at RHIC, which is indicated in Fig. 4b) as a green box. Our present results however deal with the purely gluonic sector of QCD only. For a more quantitative and meaningful comparison with RHIC results, quarks need to be incorporated in the analysis.

Let us now comment on some details of the calculations, which were not yet taken into account and should be included later. One important fact is that the lattice data for the monopole density we used actually include all magnetically charged particles, including dyons. Their electric charge would lead to additional Rutherford-like scattering which we have ignored.

In the calculation above we have not included Bose enhancement for scattered gluons. This effect adds the factor $\left(1+f\left(p^{\prime}\right)\right)$ for $g m$ and $\left(1+f\left(p_{1}^{\prime}\right)\right)\left(1+f\left(p_{2}^{\prime}\right)\right)$ for $g g$, with the prime marking the secondary gluons. If the gluon mass is small (high $T$ ) those corrections are small: their magnitude is $\langle f\rangle \sim(T / m) \sim 1 / e(T) \gg 1$. In the experimentally relevant region, when $m / T=O(1)$, the effect is not enhanced and is additionally suppressed by the expectation values of the Polyakov lines $\sim\langle L\rangle^{2}$.

\section{Acknowledgments}

We thank Jinfeng Liao and Massimo D'Elia for useful discussions. The work is partially supported by the US-DOE grants DE-FG02-88ER40388 and DE-FG03-97ER4014 and by the DFG grant SFB-TR/55.

\section{References}

[1] J. Liao and E. Shuryak, Phys. Rev. C 75, 054907 (2007). 
[2] M. N. Chernodub and V. I. Zakharov, Phys. Rev. Lett. 98, 082002 (2007) [arXiv:hep-ph/0611228].

[3] J. Liao and E. Shuryak, arXiv:0804.0255 [hep-ph].

[4] A. D’Alessandro and M. D'Elia, Nucl. Phys. B 799, 241 (2008).

[5] C. Ratti and E. Shuryak, Phys. Rev. D 80, 034004 (2009) [arXiv:0811.4174 [hep-ph]].

[6] C.Ratti, arXiv: 0907.4353 [hep-ph].

[7] M. Lublinsky, C. Ratti, E. Shuryak, arXiv: 0910.1067 [hep-ph].

[8] T. A. De Grand, D. Toussaint, Phys, Rev. D 22, 2478 (1980).

[9] J. Liao and E. Shuryak, arXiv:0804.4890 [hep-ph].

[10] P. N. Meisinger, M. C. Ogilvie and T. R. Miller, Phys. Lett. B 585, 149 (2004) [arXiv:hep-ph/0312272].

[11] E. M. Ilgenfritz, K. Koller, Y. Koma, G. Schierholz, T. Streuer, V. Weinberg and M. Quandt, PoS LAT2007, 311 (2007) [arXiv:0710.2607 [hep-lat]].

[12] H. Georgi and S. L. Glashow, Phys. Rev. D 6, 2977 (1972).

[13] D. G. Boulware, L. S. Brown, R. N. Cahn, S. D. Ellis and C. k. Lee, Phys. Rev. D 14, 2708 (1976).

[14] P. N. Meisinger, T. R. Miller, and M. C. Ogilvie, Phys. Rev. D 65, 034009 (2002); P. N. Meisinger, M. C. Ogilvie and T. R. Miller, Phys. Lett. B 585, 149 (2004); K. Fukushima, Phys. Lett. B 591, 277 (2004); C. Ratti, M. A. Thaler and W. Weise, Phys. Rev. D73, 014019 (2006); S. Roessner, C. Ratti and W. Weise, Phys. Rev. D75, 034007 (2007).

[15] J. S. Schwinger, K. A. Milton, W. y. Tsai, L. L. . DeRaad and D. C. Clark, Annals Phys. 101, 451 (1976).

[16] Y. Kazama, C. N. Yang and A. S. Goldhaber, Phys. Rev. D 15, 2287 (1977). 